\title{
CFD Analysis of the Transonic Flow over a NACA 0012 Airfoil
}

\section{Analyse CFD de l'écoulement transsonique autour d'un profil d'aile NACA 0012}

\author{
R. El Maani ${ }^{1}$, B. Radi ${ }^{2}$, A. El Hami ${ }^{3}$ \\ ${ }^{1}$ LSMI, ENSAM Meknès, Marjane 2, Meknès, Morocco, INSA de Rouen, France, rabii.el_maani@insa-rouen.fr \\ 2'LIMII, FST Settat, Maroc, bouchaib.radi@yahoo.fr \\ ${ }^{3}$ LMN, INSA de Rouen, France, abdelkhalak.elhami@insa-rouen.fr
}

\begin{abstract}
Computational Fluid Dynamics (CFD) incorporates mathematical relations and algorithms to analyze and solve the problems regarding fluid flow. CFD analysis of an airfoil produces results such as lift and drag forces which determines the ability of an airfoil. In this paper a transonic flow will be modelled over a NACA 0012 airfoil for which experimental data has been published, so that a comparison can be made. The flow to be considered is compressible and turbulent and the solver used is the density based implicit solver, which gives good results for high speed compressible flows. The results show that the predicted lift, drag and pressure cœefficients are in good agreement with experimental data. KEYWORDS. CFD, aerodynamic, NACA 0012, pressure cœfficient, airfoil.
\end{abstract}

\section{Introduction}

The rapid evolution of computational fluid dynamics (CFD) has been driven by the need for faster and more accurate methods for the calculations of flow fields around configurations of technical interest. In the past decade, CFD was the method of choice in the design of many aerospace, automotive and industrial components and processes in which fluid or gas flows play a major role. In the fluid dynamics, there are many commercial CFD packages available for modeling flow in or around objects. The computer simulations show features and details that are difficult, expensive or impossible to measure or visualize experimentally. When simulating the flow over airfoils, transition from laminar to turbulent flow plays an important role in determining the flow features and in quantifying the airfoil performance such as lift and drag. Hence, the proper modeling of transition, including both the onset and extent of transition will definitely lead to a more accurate drag prediction. The first step in modeling a problem involves the creation of the geometry and the meshes with a preprocessor. The majority of time spent on a CFD project in the industry is usually devoted to successfully generating a mesh for the domain geometry that allows a compromise between desired accuracy and solution cost. After the creation of the grid, a solver is able to solve the governing equations of the problem. The basic procedural steps for the solution of the problem are the following. First, the modeling goals have to be defined and the model geometry and grid are created. Then, the solver and the physical models are stepped up in order to compute and monitor the solution. Afterwards, the results are examined and saved and if it is necessary we consider revisions to the numerical or physical model parameters.

\section{Mathematical formulation}

For the description of fluid flows usually the Eulerian formulation is employed, because we are usually interested in the properties of the flow at certain locations in the flow domain. We restrict ourselves to the 
case of linear viscous isotropic fluids known as Newtonian fluids, which are by far the most important ones for practical applications. Newtonian fluids are characterized by the following material law for the Cauchy stress tensor $T$ :

$$
T_{i j}=\mu\left(\frac{\partial v_{i}}{\partial x_{j}}+\frac{\partial v_{j}}{\partial x_{i}}-\frac{2}{3} \frac{\partial v_{k}}{\partial x_{k}} \delta_{i j}\right)-p \delta_{i j}
$$

with the velocity vector $v_{i}$ with respect to Cartesian coordinate $x_{i}$, the pressure $p$, the dynamic viscosity $\mu$, and the Kronecker symbol $\delta_{i j}$. The Navier-Stokes equations of incompressible flows may be written on the spatial fluid mechanics domain as :

$$
\begin{aligned}
\frac{(\partial v)}{\partial t}+\nabla \cdot(\rho v \otimes v-\sigma)-\rho f & =0 \\
\nabla \cdot v & =0
\end{aligned}
$$

where $\rho, v$, and $f$ are the density, velocity, and the external force, respectively, and $\sigma$ the stress tensor is defined as :

$$
\sigma(v, p)=-p I+2 \mu \varepsilon(v)
$$

Here $p$ is the pressure, $I$ is the identity tensor, $\mu$ is the dynamic viscosity, and $\epsilon(v)$ is the strain-rate tensor given by :

$$
\epsilon(v)=\frac{1}{2}\left(\nabla v+\nabla v^{T}\right)
$$

The boundary conditions associated to the fluid domain are :

$$
\left.v\right|_{\Gamma_{v}}=\bar{v}
$$

where $\bar{v}$ can be a known velocity profile at the boundary.

\section{Turbulence model}

In general, turbulence models attempt to modify the original unsteady Navier-Stokes equations by introducing averaged and fluctuating quantities to produce Reynolds Averaged Navier-Stokes equations (RANS). The turbulence models based on the RANS equations are known as statistical turbulence models because of the statistical mean procedure used to obtain the equations. In our study the k-Omega SST model will be used [3]. 


\subsection{K-Omega SST model}

The shear-stress transport (SST) $k-\omega$ model was developed by Menter [9] to effectively blend the robust and accurate formulation of the model in the near-wall region with the freestream independence of the $k-\omega$ model in the far field.

The SST $k-\omega$ model has a similar form to the standard $k-\omega$ model :

$$
\begin{aligned}
& \frac{\partial}{\partial t}(\rho k)+\frac{\partial}{\partial x_{i}}\left(\rho k u_{i}\right)=\frac{\partial}{\partial x_{j}}\left(\Gamma_{k} \frac{\partial k}{\partial x_{j}}\right)+\widetilde{G_{k}}-Y_{k}+S_{k} \\
& \frac{\partial}{\partial t}(\rho \omega)+\frac{\partial}{\partial x_{i}}\left(\rho \omega u_{i}\right)=\frac{\partial}{\partial x_{j}}\left(\Gamma_{\omega} \frac{\partial \omega}{\partial x j}\right)+G_{\omega}-Y_{\omega}+D_{\omega}+S_{\omega}
\end{aligned}
$$

Where $D_{\omega}$ represents the cross-diffusion term. The turbulent viscosity $\mu_{i}$ is computed as follows :

$$
\mu_{t}=\frac{\rho k}{\omega} \frac{1}{\max \left[\frac{1}{\alpha^{*}}, \frac{S F_{2}}{a_{1} \omega}\right]}
$$

Where $: \sigma_{k}=\frac{1}{\frac{F_{1}}{\sigma_{k, 1}}+\frac{\left(1-F_{1}\right)}{\sigma_{k, 2}}}$ and $\sigma_{\omega}=\frac{1}{\frac{F_{1}}{\sigma_{\omega, 1}}+\frac{\left(1-F_{1}\right)}{\sigma_{\omega, 2}}} \cdot F_{1}$ and $F_{2}$ are the blending functions,

The term $\widetilde{G}_{k}$ represents the production of turbulence kinetic energy, and is defined as :

$$
\widetilde{G_{k}}=\min \left(G_{k}, 10 \rho \beta^{*} k \omega\right)
$$

The term $G_{\omega}$ represents the production of and is given by :

$$
G_{\omega}=\frac{\alpha}{\nu_{t}} G_{k}
$$

The SST $k-\omega$ model is based on both the standard $k-\omega$ model and the standard $k-\epsilon$ model. To blend these two models together, the standard $k-\epsilon$ model has been transformed into equations based on $k$ and $\omega$, which leads to the introduction of a cross-diffusion term $D_{\omega}$ is defined as :

$$
D_{\omega}=2\left(1-F_{1}\right) \rho \sigma_{\omega, 2} \frac{1}{\omega} \frac{\partial k}{\partial x_{j}} \frac{\partial \omega}{\partial x_{j}}
$$

Model constants are :

$$
\begin{array}{rlrl}
\sigma_{k, 1} & =1.176, \quad \sigma_{\omega, 1}=2.0, & \sigma_{k, 2} & =1.0, \quad \sigma_{\omega, 2}=1.168 \\
a_{1} & =0.31, \quad \beta_{i, 1}=0.075, \quad \beta_{i, 2} & =0.0828, \quad k=0.41
\end{array}
$$




\section{Numerical simulation}

\subsection{Problem statement}

In this paper, the NACA 0012, the well documented airfoil from the 4-digit series of NACA airfoils, was utilized. The NACA 0012 airfoil is symmetrical; the 00 indicates that it has no camber. The 12 indicates that the airfoil has a $12 \%$ thickness to chord length ratio; it is $12 \%$ as thick as it is long. Mach number for the simulations was evaluated for $\mathrm{M}=0.5$ and $\mathrm{M}=0.7$, same with the reliable experimental data from T.J. Coakley (1987) [9], in order to validate the present simulation. The free stream total temperature is $311 \mathrm{~K}$, which is the same as the environmental temperature. The density of the air at the given temperature is $\rho=1.225 \mathrm{~kg} / \mathrm{m}^{3}$ and the viscosity is $\mu=1.7894 \times 10^{-5} \mathrm{~kg} / \mathrm{m}$.s. For this Mach numbers, the flow can be described as compressible. A segregated, implicit solver was utilized (ANSYS/Fluent). Calculations were done for angle of attack $\alpha=1.55^{\circ}$. The airfoil profile meshes were created using structured meshes, which consist of a variety of quadrilateral elements. The resolution of the mesh was greater in regions where greater computational accuracy was needed, such as the region close to the airfoil.

The first step in performing a CFD simulation should be to investigate the effect of the mesh size on the solution results. Generally, a numerical solution becomes more accurate as more nodes are used, but using additional nodes also increases the required computer memory and computational time. The appropriate number of nodes can be determined by increasing the number of nodes until the mesh is sufficiently fine so that further refinement does not change the results.

\subsection{Boundary conditions}

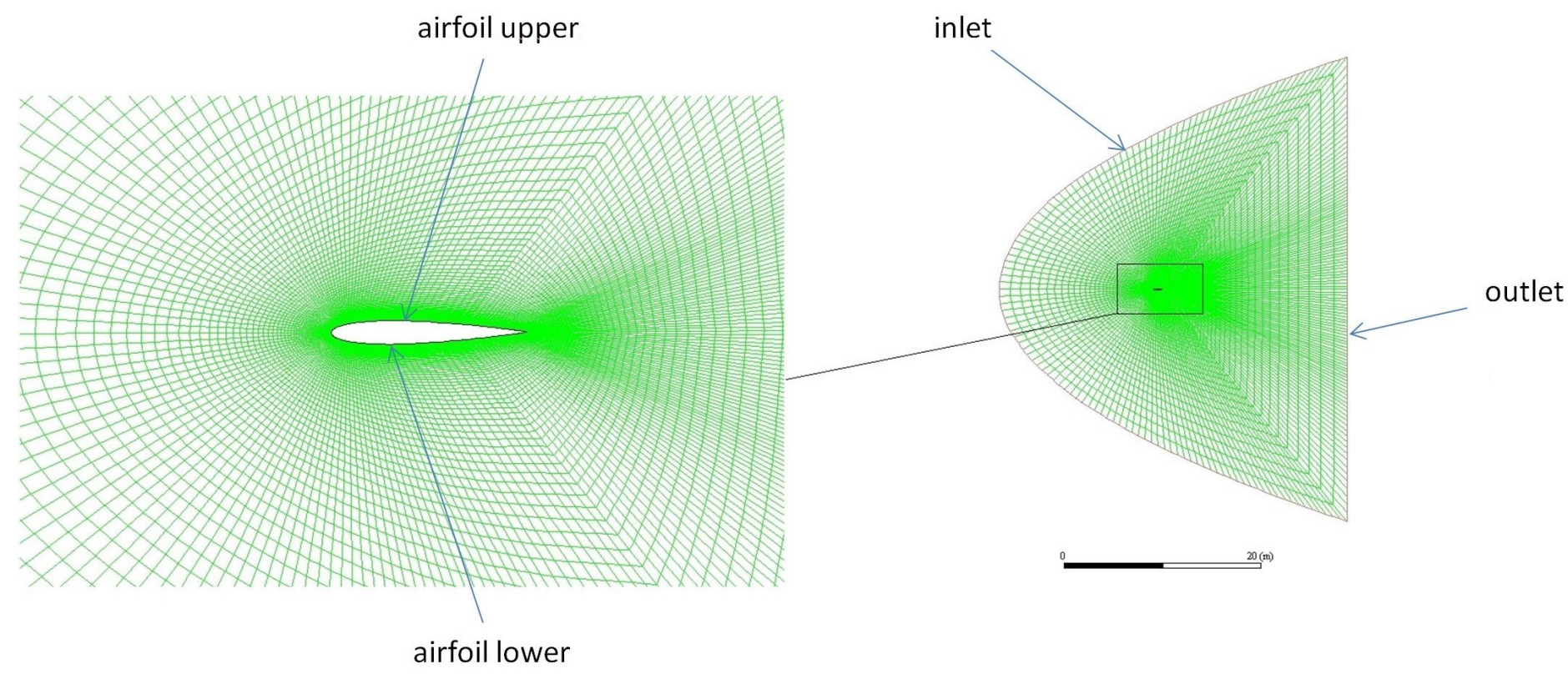

Figure 1. Boundary conditions

- airfoil_lower and airfoil_upper : We will be setting this to a wall boundary condition, setting the velocity there to be 0 . 
- inlet, outlet : We will be setting far-field pressure, Mach number, temperature, and components of the velocity. This allows for the calculation of the speed of sound, and the velocity direction.

\subsection{Results}

The main source of experimental data, used here for comparison, are the measured flow parameters on straight wings, with unchanged cross sections along their span, and collected in the Experimental Data Base for Computer Program Assessment (AGARD-AR-138, 1979) for checking computational fluid dynamic methods. This $12 \%$ thick symmetrical airfoil is widely used for testing different computing methods. The data on the flow over the airfoil are obtained in several wind tunnels, but, according to Hoist (1987) [10], it is best to take the data from Harris's tests in the Langley 8-Foot Transonic Pressure Tunnel [11]. Computations were performed at a Reynolds number of $\operatorname{Re}=9.10^{6}$. Comparison of the computed results with Harris's data for the pressure cœefficient are shown in Figures 3 and 5 at $\mathrm{M}=0.5$ and $\mathrm{M}=0.7$ at angle of attack $\alpha=1.55^{\circ}$. Table 1 pressents a comparision of the lift and drag coefficients with the experimental data.
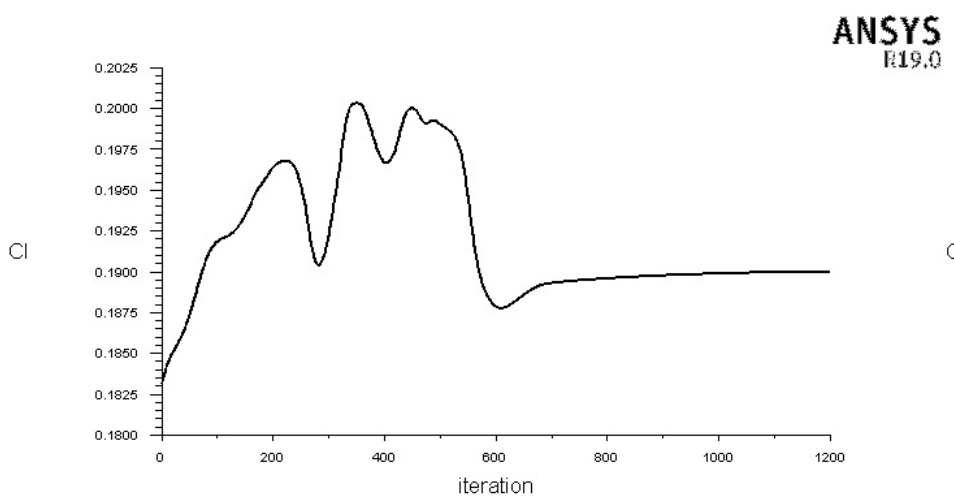

ANSYS

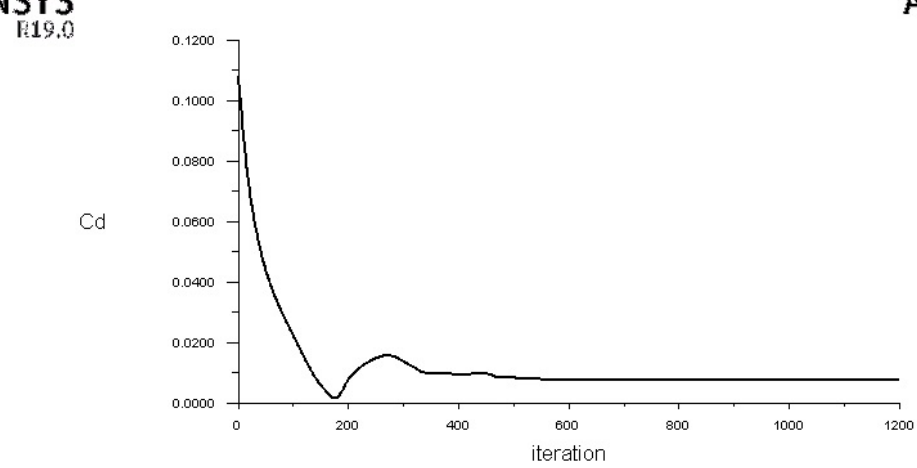

Figure 2. Evolution of the drag and lift coefficients for Mach 0.5

- airfoil_lower

- airfoil_upper

- Experimental-lower

- Experimental-upper
Pressure Coefficient
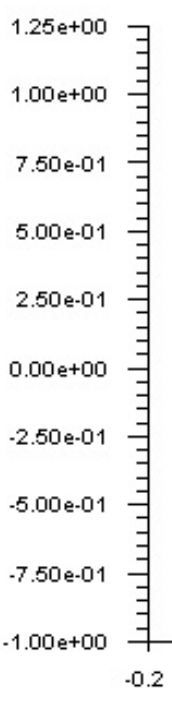

ANSYS

Fis.0

Figure 3. $C_{p}$ along the upper and lower airfoil surfaces for Mach 0.5 

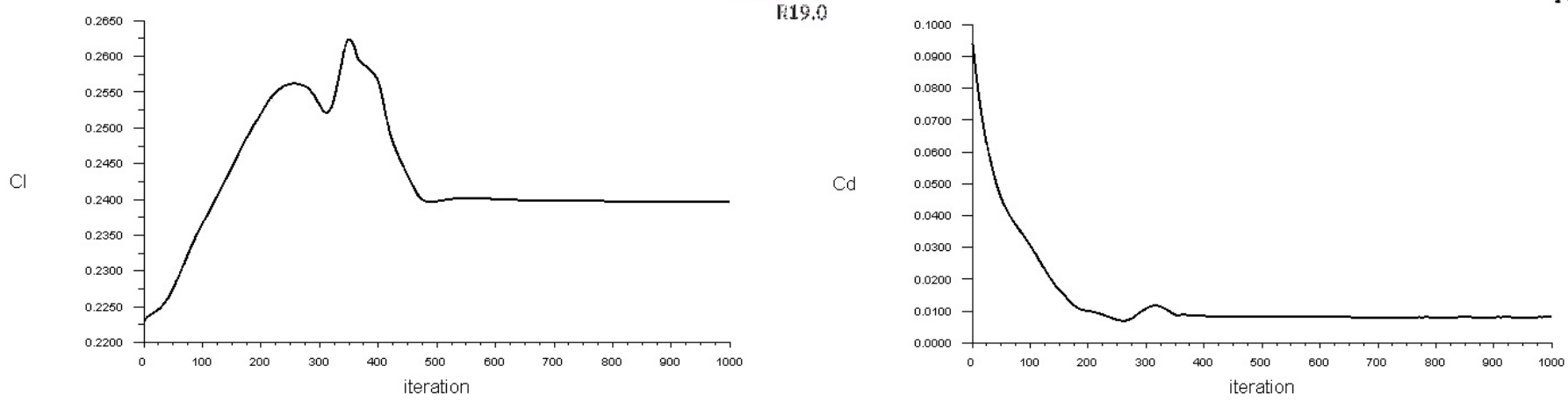

Figure 4. Evolution of the drag and lift coefficients for Mach 0.7

- airfoil_lower

- airfoil_upper

- Experimental-lower

- Experimental-upper

Pressure Coefficient

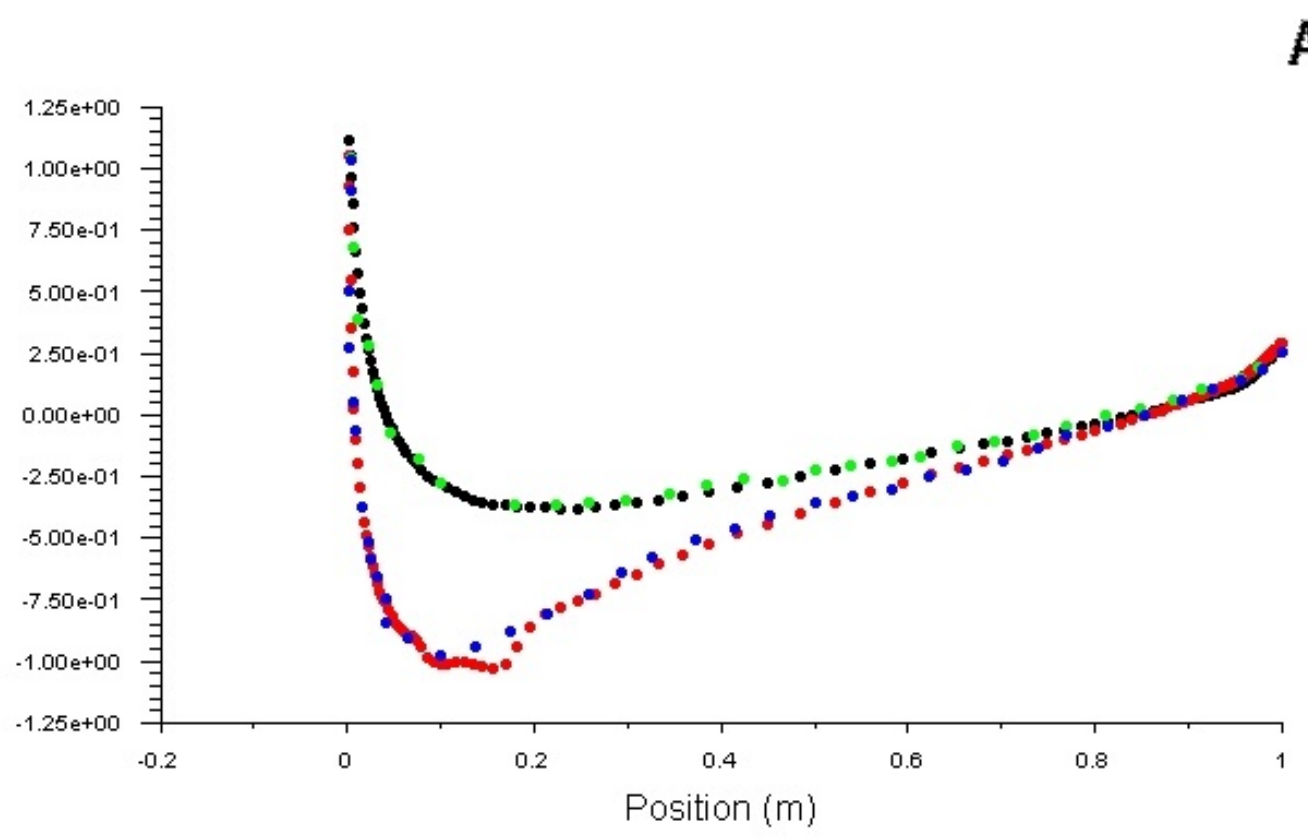

Figure 5. $C_{p}$ along the upper and lower airfoil surfaces for Mach 0.7
ANSYS

F19.0

\begin{tabular}{|c|c|c|c|} 
& Experimental data & Mach 0.5 & Mach 0.7 \\
\hline $\mathrm{C}_{l}$ & 0.241 & 0.190 & 0.239 \\
$\mathrm{C}_{d}$ & 0.0079 & 0.0079 & 0.0081 \\
\hline
\end{tabular}

Tableau 4.1. Comparison of the drag $\left(C_{d}\right)$ and lift $\left(C_{l}\right)$ coefficients

Figures 3 and 5 shows experimental and computed pressure distributions with experimental and computational angle of attack of $1.55^{\circ}$, at a Mach number of 0.5 and 0.7 , respectively. In the second case $(\mathrm{M}=0.7)$ there is no separation, and the computed and experimental pressure distributions are in close agreement with one another. The computations do, however, indicate the presence of a weak shock wave near the chordwise Iocatlon of $\mathrm{x} / \mathrm{c}=0.15$; the shock is not apparent in the experimental pressure distrlbutions. In the first case a clear difference can be seen between the simulated and the experimental data especially at the upper airfoil. The computed lift and drag coefficients for those cases are compared with experimental normal force and drag coefficients in Table 1. It is found that the second case gives lift and drag coefficients that are slightly the same with the experimental values. 


\section{Conclusion}

In this paper ANSYS/FLUENT has been used within a workbench project to compute the transonic, compressible flow over a NACA0012 airfoil. The implicit density based solver with solution steering was employed and the computed results have been compared to published experimental data and good agreement was achieved. A case comparison has been carried out within CFD Post to compare the pressure fields at Mach 0.5 and Mach 0.7.

\section{Bibliographie}

[1] El Hami A., RADi B., Fluid-Structure Interactions and Uncertainties : Ansys and Fluent Tool, John Wiley and Sons, 2017.

[2] El MaAni R., Étude Basée Sur l'optimisation Fiabiliste En Aérodynamique, INSA de Rouen, 2016.

[3] Launder B. E., Spalding D. B., Lectures in Mathematical Models of Turbulence, Academic Press, London, England, 1972.

[4] Souli M., Benson D. J., Arbitrary Lagrangian-Eulerian and Fluid-Structure Interaction, ISTE Ltd and John Wiley Sons, 2010.

[5] HuAng S., Li R., Li Q.S., Numerical simulation on fluid-structure interaction of wind around super-tall building at high reynolds number conditions, Structural Engineering and Mechanics, 46(2) : 197-212, 2013.

[6] Patankar S.V., Numerical Heat Transfer and Fluid Flow, Hemisphere Publishing, New York, USA, 1980.

[7] Ferziger J.H., Peric M., Computational Methods for Fluid Dynamics, Springer, Berlin, Germany, 1996.

[8] Chopra A., Dynamics of Structures, Pearson Prentice Hall, 2nd ed, 2001.

[9] CoAKley T.J., Numerical Simulation of Viscous Transonic Airfoil Flows, NASA Ames Research Center, AIAA-870416, 1987.

[10] HoIST T.L., Viscous transonic airfoil workshop compedium of results, AIAA Paper No. 87-1460, 1987.

[11] HARRIS C.D., Two-Dimensional Aerodynamic Characteristics of the NACA 0012 Airfoil in the Langley 8-foot Transonic Pressure Tunnel, NASA Ames Research Center, NASA TM 81927, 1981. 